\title{
RESONATING SILICON BEAM FORCE SENSOR
}

F. R. BLOM, S. BOUWSTRA, J. H. J. FLUITMAN and M. ELWENSPOEK

Department of Electrical Engineering, University of Twente, P. O. Box 217, 7500 AE

Enschede (The Netherlands)

\begin{abstract}
A resonating silicon-beam force sensor is being developed using micromachining of silicon and IC-compatible processes. Results are reported here of measurements on the force-to-frequency transfer of bare silicon prototypes. The measurements with forces on the sensor beam up to $0.4 \mathrm{~N}$ show a frequency shift of 3.1 to 5.2 times the unloaded resonance frequency $f_{0}\left(f_{0} \simeq\right.$ 3 to $5 \mathrm{kHz}$ ), depending on the exact dimensions. Considering these figures, we can predict a frequency shift of 18.3 to $27.6 \mathrm{kHz}$ at the maximum load of $1.0 \mathrm{~N}$ for the measured samples. Due to the sample lay-out, a force transfer is present from the externally applied force to the actual pulling force on the sensor beam. Using a simple model to calculate this reduction, we obtain good agreement between the measurements and predictions.
\end{abstract}

Introduction

The principle of the resonating beam as a force sensor has often been demonstrated in the literature. Examples are the vibrating-wire transducer $[1,2]$ and the double-ended tuning fork quartz resonator [3-5]. The sensor under development uses silicon for the mechanical material and a thin layer of piezoelectric $\mathrm{ZnO}$ as excitation and detection material. The working principle is similar to that reported for the resonating membrane pressure sensor [6]. Such a silicon force sensor can be used in electronic precision weighing scales having a range up to $1 \mathrm{~N}$ and with a resolution of better than 1 part per 10000.

In this paper results are reported of the relation between the pulling force and the resonance frequency of a bare silicon prototype of this force sensor. The lay-out used is shown in Fig. 1. For safe manipulation, the actual sensor beam is supported by two bars of the original wafer thickness. This gives rise to a force reduction. By choosing the proper dimensions, a large frequency shift can be achieved of the order of several times the unloaded resonance frequency, leading to a high resolution. 


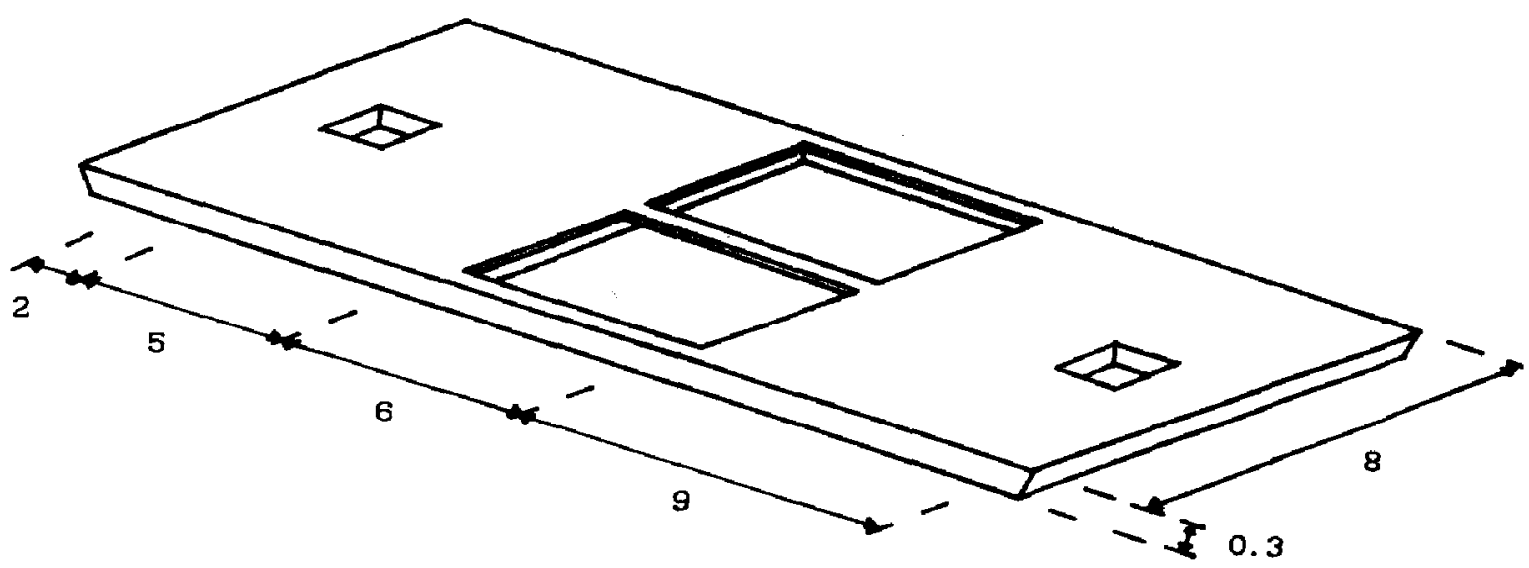

Fig. 1. Lay-out of the silicon force sensor with some characteristic dimensions (in $\mathrm{mm}$ ).

\section{Force-frequency relation}

The differential equation of vibrations of a pulled beam clamped at both ends (see e.g., [7]) can be solved analytically, resulting in an implicit formula for the relation between the resonance frequency and the pulling force. This relation can be approximated for small forces by

$f \simeq f_{0}\left\{1+0.3\left[\frac{l}{d}\right]^{2} \frac{F}{E b d}\right\}^{1 / 2}$

with $f$ the loaded resonance frequency, $f_{0}$ the unloaded resonance frequency, $F$ the pulling force, $E$ the Young's modulus, $l$ the length of the beam, $d$ its thickness and $b$ its width.

For a beam with dimensions $l=6.0 \mathrm{~mm}, b=0.4 \mathrm{~mm}$ and $d=20 \mu \mathrm{m}$, eqn. (1) deviates $1 \%$ from the exact solution for a force of $0.05 \mathrm{~N}$, a failure that increases rapidly for larger forces. However, eqn. (1) is still useful for calculating estimates of the sensor properties, such as its sensitivity. The calculated frequency shift for the sensors with the dimensions considered can be as high as 4 to 12 times the unloaded resonance frequency $f_{0}\left(f_{0} \simeq 3\right.$ to $5 \mathrm{kHz}$ ).

\section{Force transfer}

In order to calculate the transfer of the externally applied force $F_{\text {ex }}$ to the actual pulling force $F$ on the sensor beam, we first consider the in-plane transfer. For this we approximate the frame of the sample by the simple beam structure shown in Fig. 2. Here it can be seen that the displacement of the end of the sensor beam consists of two parts: the displacement $u_{0}$ of the support bars due to the homogeneous force, and the deflection $u_{1}$ of the simply supported beam in its plane, due to bending and shearing. In the 


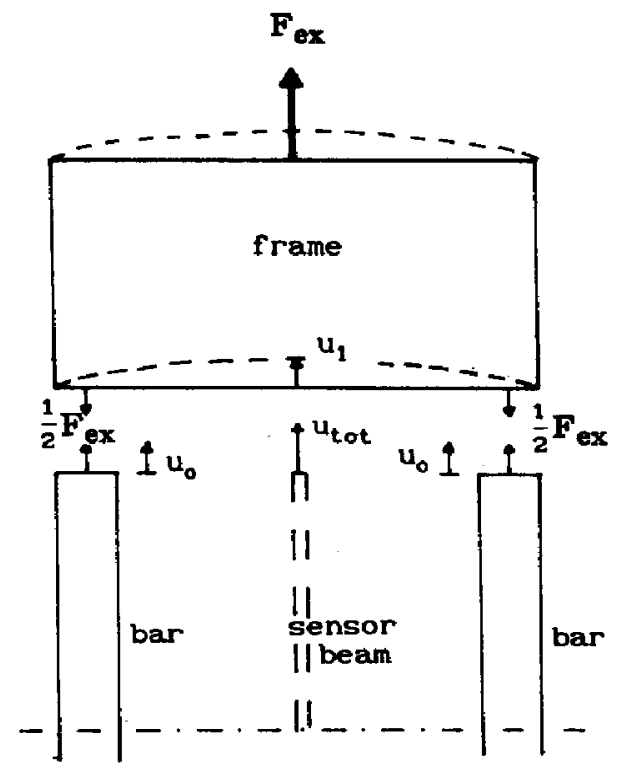

Fig. 2. Displacements related to the in-plane force transfer from external force to actual force on the sensor beam. Part of the sample is shown in top-view.
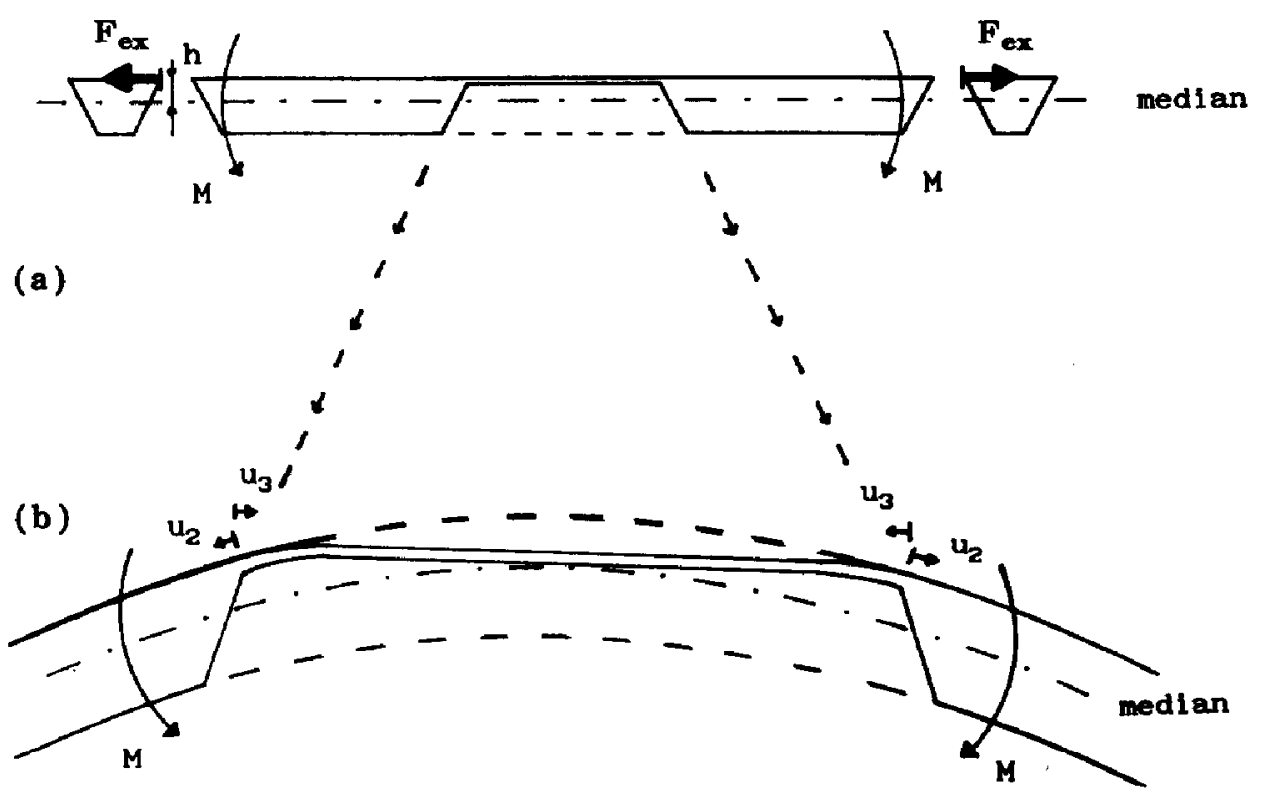

Fig. 3. Effect of the bending moment due to distance $h$ between the median of the structure and the point of force action: (a) side-view of the sample; (b) displacements related to the moment. The dotted lines show the support bars.

calculation it is assumed that the stress distribution fits properly with this mechanical problem.

Another effect we have to account for is the bending of the sample. Because the external force acts above the median of the sample, a bending moment arises (see Fig. 3). This moment on the bars results in two effects: 
the sensor beam will be stretched because it lies above the median (displacement $u_{2}$ ), but it will also be compressed because the end points of the bars move closer to each other due to the bending (displacement $u_{3}$ ). In this case the flexural rigidity of the sensor beam in neglected. The elongation of the sensor beam due to its own deflection is negligible, i.e., the sensor beam can be considered as a ribbon.

The total elongation of the sensor beam can now be composed of the separate effects. The solution to this problem shows that $u_{0}, u_{1}$ and $u_{2}$ are positive and proportional to the external force, whereas $u_{3}$ is negative and proportional to the square of this force. Hence the force transfer itself is force dependent.

\section{Experiments}

The samples are made by anisotropic wet chemical etching of silicon using a KOH solution. All the sensor beams are $6 \mathrm{~mm}$ long and $0.4 \mathrm{~mm}$ wide; the thickness ranges from 12 to $30 \mu \mathrm{m}$. The support beams are of the original wafer thickness, i.e., $285 \mu \mathrm{m}$, and the average width is $380 \mu \mathrm{m}$.

The experimental set-up is shown in Fig. 4. The beam is driven acoustically by means of a loudspeaker and the vibration is detected optically using a Michelson interferometer. A network analyzer is used to determine the

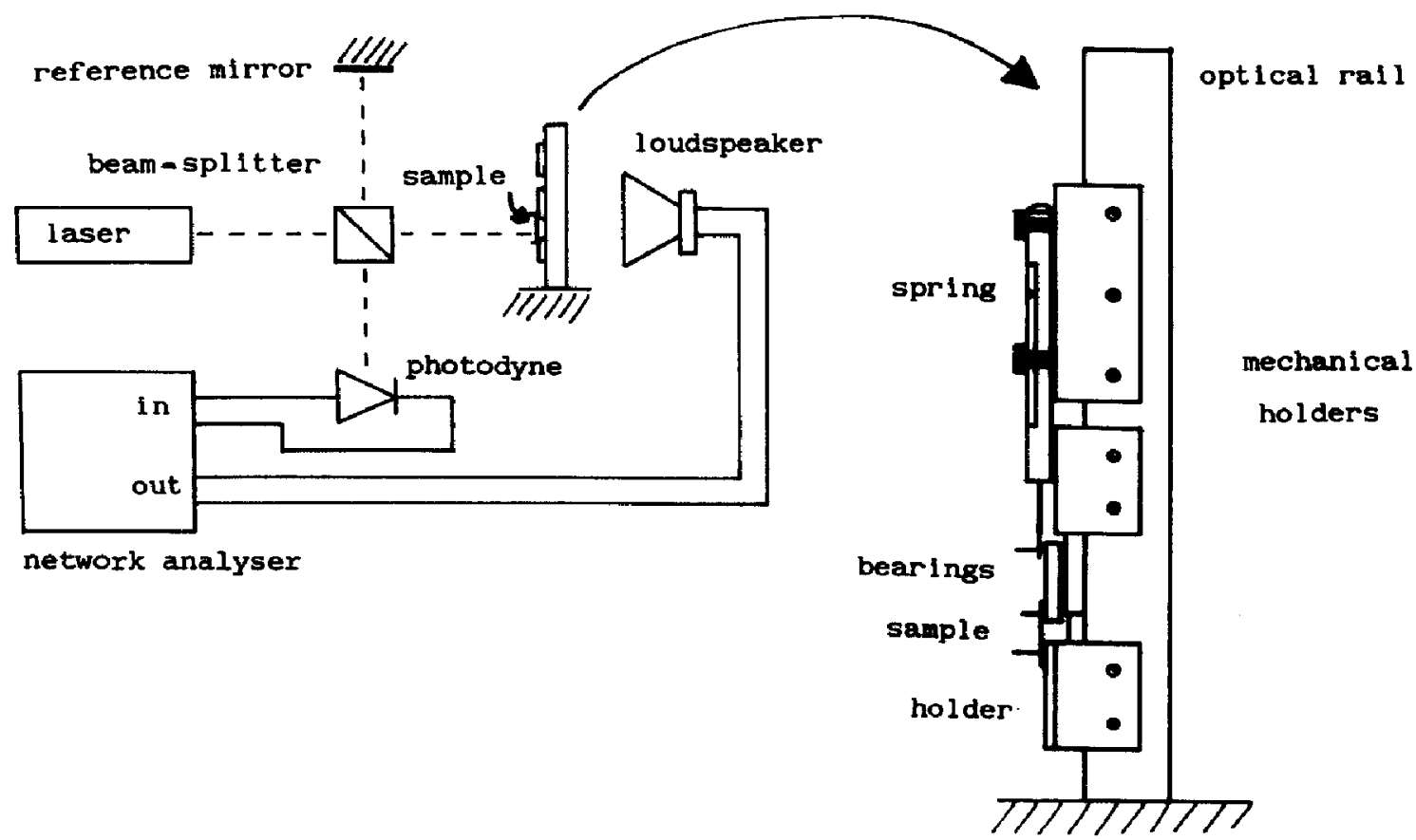

Fig. 4. Experimental set-up with a close-up of force-introducing system with the epring and bearings. 
transfer function of the sensor. The external force is applied by means of a spring and bearings. The springs that are used have maximum loads of 3 and $10 \mathrm{~N}$, respectively.

Results and discussion

Results of measurements are shown in Fig. 5 for two beams with thicknesses of $13 \mu \mathrm{m}$ and $23 \mu \mathrm{m}$ respectively. The error in the external applied load is $0.05 \mathrm{~N}$, while that in the frequency is smaller than $0.1 \%$. In the same Figure the predicted curves are also drawn, using the exact solution of the force-frequency transfer and the calculated reduction factor. Because the thickness of the sensor beams is only known within 5\%, a large error occurs in the theoretical simulation. The worst-case curves are also plotted in Fig. 5, where we see that the measurements fit well between these curves.

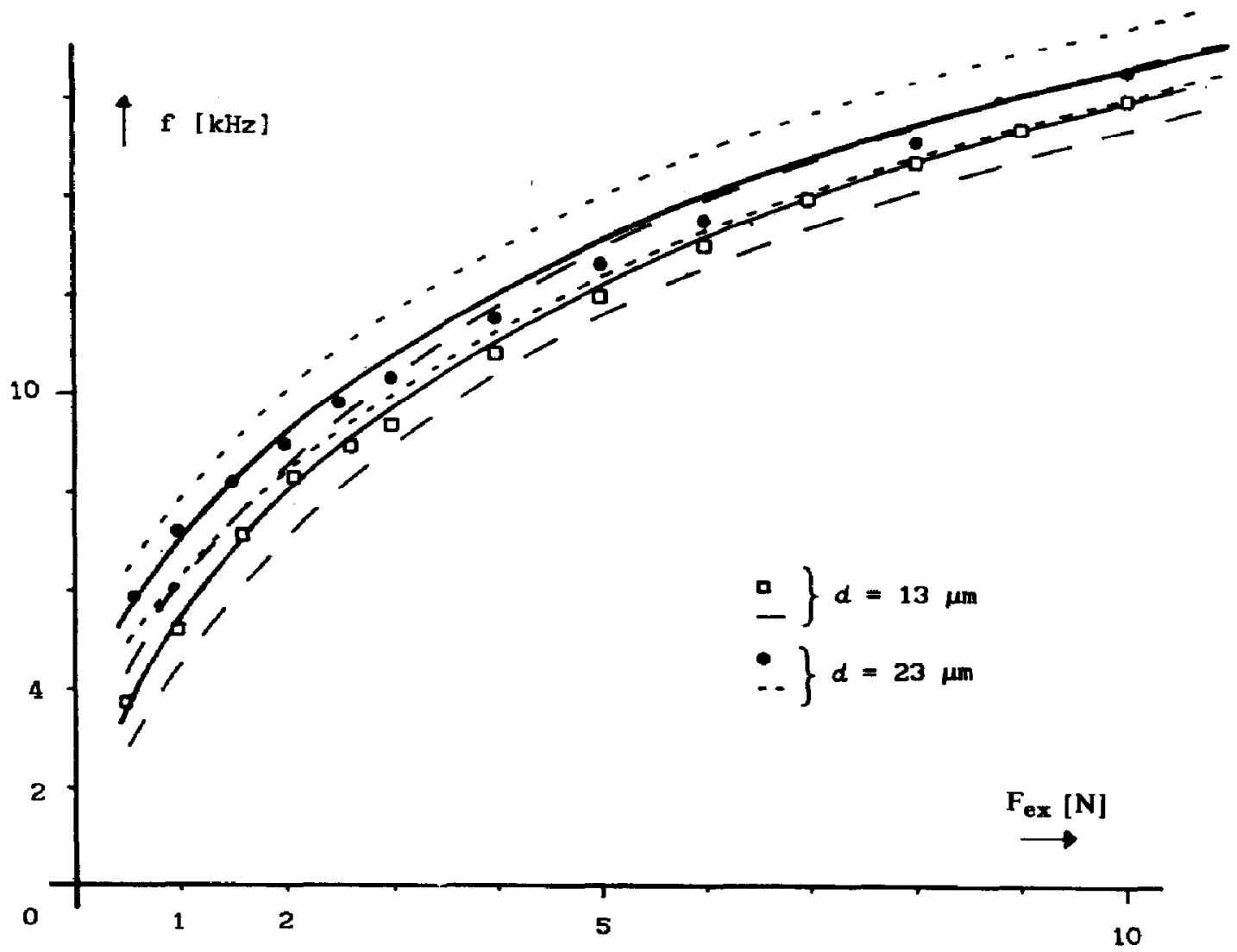

Fig. 5. Graph of the frequency as a function of the force on the spring for beams with thicknesses of $13 \mu \mathrm{m}$ and $23 \mu \mathrm{m}$. Also shown are theoretical curves, with the four dashed ones being the worst-case curves. 
We also examined the flatness of the sample by projecting its interference pattern. The predicted bending of the support bars was observed, whereas the sensor beam remained relatively flat.

The approximation used for the force reduction factor is sufficiently accurate to predict the frequency shift of the sensor. Recalculations from Fig. 5 give an $f / f_{0}$ of 5.2 for a force of $0.25 \mathrm{~N}$ applied to the sensor beam with a thickness of $13 \mu \mathrm{m}$. Using eqn. (1), we predict an $f / f_{0}$ of 10.2 when the maximum force of $1.0 \mathrm{~N}$ acts on the sensor beam, which corresponds to a frequency shift of $27.6 \mathrm{kHz}$.

\section{Conclusions}

The results demonstrate that it is possible to construct a force sensor from silicon wafers using micro-machining and IC-compatible techniques, which is suitable for use in precision weighing scales. The exact lay-out of the sensor must be chosen with care in view of the way the force acts on the sensor.

The measurements at forces up to $0.4 \mathrm{~N}$ show a frequency shift of 3.1 to 5.2 times the unloaded resonance frequency. This means that the predicted frequency shift of the sensor beam can be obtained at a force of $1.0 \mathrm{~N}$ in order to obtain the desired resolution of better than 1 part per 10000.

The force transfer from the external force to the actual force on the sensor beam can be approximated by solving a complex mechanical problem. Use of this reduction factor to simulate the force-frequency transfer of the sensor leads to curves that fit the measured data reasonably well. A threedimensional analysis is required to determine the exact force transfer.

\section{Acknowledgements}

The authors would like to thank John Baxter for carefully reading the manuscript. This research in the program of the Foundation for Fundamental Research on Matter (FOM) is sponsored by the Netherlands Technology Foundation (STW).

\section{References}

1 U. Erdem, Force and weight measurement, J. Phys. E: Sci. Instrum., 15 (1982) 857 - 872.

2 H. R. Zulliger, Precise measurement of small forces, Sensors and Actwators, 4 (1983) $483-495$.

3 E. P. EerNise, Miniature quartz resonator force transducer, U.S. Patent 4215570 (1980). 
4 R. G. Kirman, A vibrating quartz force sensor, Proc. Transducer Tempcon Conf., London, June $14-16,1983$, pp. $97-121$.

5 T. Ueda, F. Kohasaka and E. Ogita, Precision force transducer using mechanical resonators, Proc. 10th Conf. IMEKO TC3, preprints, Recent Advances in Weighing Technology and Force Measurements, Kobe, Japan, September $11-14,1984$, pp. 17 - 22.

6 J. G. Smits, H. A. C. Tilmans and T. S. T. Lammerink, Pressure dependence of resonant diaphragm pressure sensor, Proc. 3rd Int. Conf. Solid-State Sensors and Actuators (Transducers' 85), Philadelphia, PA, U.S.A., June $11-14,1985$, pp. 93 96.

7 S. Timoshenko, D. H. Young and W. Weaver, Jr., Vibration Problems in Engineering, Wiley, New York, 4th edn., 1974, pp. $408-431$. 